\title{
A REMARK ON GETZLER'S SEMI-CLASSICAL APPROXIMATION
}

\author{
DAN PETERSEN
}

\begin{abstract}
Ezra Getzler notes in the proof of the main theorem of "The semiclassical approximation for modular operads" that "A proof of the theorem could no doubt be given using la combinatorial interpretation in terms of a sum over necklaces]; however, we prefer to derive it directly from Theorem 2.2". In this note we give such a direct combinatorial proof using wreath product symmetric functions.
\end{abstract}

\section{INTRODUCTION}

Let $\mathcal{V}$ be a stable $\mathbb{S}$-module, i.e. a collection $\mathcal{V}((g, n))$ of representations of $\mathbb{S}_{n}$ indexed by pairs $(g, n)$ with $2 g-2+n>0$. The paper [Getzler and Kapranov 1998] defines an endofunctor $\mathbb{M}$ on the category of stable $\mathbb{S}$-modules, modeled on the way that the moduli spaces $\mathcal{M}_{g, n}$ are glued together to form boundary strata of $\overline{\mathcal{M}}_{g^{\prime}, n^{\prime}}$. In [Getzler 1998] an explicit formula is derived which describes the genus one part of $\mathbb{M} \mathcal{V}$ in terms of $\mathcal{V}$. The case of genus zero had been described already in [Getzler 1995, in terms of the Legendre transform.

If $V$ is a representation of $\mathbb{S}_{n}$, let $\operatorname{ch} V$ denote the corresponding symmetric function. The main theorem of [Getzler 1998] reads]

$$
\mathbf{b}_{1}=\left(\mathbf{a}_{1}-\frac{1}{2} \sum_{n \geq 1} \frac{\phi(n)}{n} \log \left(1-\psi_{n}\left(\mathbf{a}_{0}^{\prime \prime}\right)\right)+\frac{\dot{\mathbf{a}}_{0}\left(1+\dot{\mathbf{a}}_{0}\right)+\frac{1}{4} \psi_{2}\left(\mathbf{a}_{0}^{\prime \prime}\right)}{1-\psi_{2}\left(\mathbf{a}_{0}^{\prime \prime}\right)}\right) \circ\left(h_{1}+\mathbf{b}_{0}^{\prime}\right),
$$

where

$$
\mathbf{a}_{g}=\sum_{n} \operatorname{ch} \mathcal{V}((g, n))
$$

and

$$
\mathbf{b}_{g}=\sum_{n} \operatorname{ch} \mathbb{M} \mathcal{V}((g, n))
$$

are generating functions. For a symmetric function $f, f^{\prime}$ denotes $\frac{\partial f}{\partial p_{1}}$ and $\dot{f}$ denotes $\frac{\partial f}{\partial p_{2}}$. The $\psi_{k}$ are the Adams operations defined by $\psi_{k}(f)=p_{k} \circ f$.

Let us introduce some terminology.

Definition 1.1. A graph is a finite set with a partition and an involution, as in e.g. [Getzler and Kapranov 1998]. A corolla is a graph with one vertex. A necklace is a graph $\Gamma$ such that $b_{1}(|\Gamma|)=1$ and which is not disconnected by removing any edge.

\footnotetext{
${ }^{1}$ The term $\frac{1}{4} \psi_{2}\left(\mathbf{a}_{0}^{\prime \prime}\right)$ is missing in Getzler's paper; it was pointed out in [Consani and Faber 2006] that there is a minor computational error there.
} 
The term $\left(h_{1}+\mathbf{b}_{0}^{\prime}\right)$ can be interpreted combinatorially as taking one copy of the trivial representation, together with all possible graphs corresponding to a stable tree of genus zero vertices with a single distinguished leg. The plethysm should be interpreted as a "gluing" operation. In the larger expression

$$
\left(\mathbf{a}_{1}-\frac{1}{2} \sum_{n \geq 1} \frac{\phi(n)}{n} \log \left(1-\psi_{n}\left(\mathbf{a}_{0}^{\prime \prime}\right)\right)+\frac{\dot{\mathbf{a}}_{0}\left(1+\dot{\mathbf{a}}_{0}\right)+\frac{1}{4} \psi_{2}\left(\mathbf{a}_{0}^{\prime \prime}\right)}{1-\psi_{2}\left(\mathbf{a}_{0}^{\prime \prime}\right)}\right)
$$

the first term describes corollas of genus one, and the claim is that the rest is the sum over all possible graphs that are given by a necklace of genus zero vertices. Then the plethysm with $\left(h_{1}+\mathbf{b}_{0}^{\prime}\right)$ gives us the sum over all graphs obtained by attaching genus zero trees (possibly empty, corresponding to the trivial representation) to either the genus one vertex or a necklace, which produces a sum over all stable graphs of genus one, and we recover the definition of $\mathbb{M}$.

Hence the meat of the theorem lies in showing that

$$
-\frac{1}{2} \sum_{n \geq 1} \frac{\phi(n)}{n} \log \left(1-\psi_{n}\left(\mathbf{a}_{0}^{\prime \prime}\right)\right)+\frac{\dot{\mathbf{a}}_{0}\left(1+\dot{\mathbf{a}}_{0}\right)+\frac{1}{4} \psi_{2}\left(\mathbf{a}_{0}^{\prime \prime}\right)}{1-\psi_{2}\left(\mathbf{a}_{0}^{\prime \prime}\right)}
$$

gives exactly the sum over necklaces of genus zero vertices. It is pointed out in the proof of the theorem that there probably exists a direct combinatorial proof of this fact. However, Getzler deduces it by somewhat involved computations using the more general Getzler-Kapranov formula of [Getzler and Kapranov 1998] which relates $\mathcal{V}$ and $\mathbb{M} \mathcal{V}$ for all $g$ and $n$, and an explicit representation of the so-called plethystic Laplacian in terms of a formal heat kernel over $\Lambda((\hbar))$.

In this note we give a combinatorial proof of the fact that the sum over necklaces gives exactly this expression, using only standard facts about wreath product symmetric functions. In particular we are able to give a combinatorial interpretation to the terms in the sum: the first is a sum over all rotational symmetries of the necklaces, and the second is a sum over all symmetries under reflection.

Convention. We consider throughout $\mathbb{S}$-modules and representations in some fixed symmetric monoidal category $\mathcal{E}$ with finite colimits, additive over a field of characteristic zero. The final assumption allows us to identify $\mathbb{S}$-modules in $\mathcal{E}$ with symmetric functions, i.e. $K_{0}([\mathbb{S}, \mathcal{E}]) \cong K_{0}(\mathcal{E}) \widehat{\otimes} \Lambda$, where $\Lambda$ is the ring of symmetric functions graded by degree, and $\widehat{\otimes}$ is the completed tensor product. We tacitly omit $\mathcal{E}$ from the notation.

\section{CyClically ordered NeCKLaces}

We start by considering the easier case of necklaces which are equipped with a cyclic ordering. This case is used in the article [Petersen 2012], and it will serve as motivation for the proof in the unordered case.

Definition 2.1. An $\mathbb{S}$-module $\mathcal{V}$ is the data of a representation $\mathcal{V}(n)$ of $\mathbb{S}_{n}$ for each positive integer $n$. (Usually one would include $n=0$, but it will be slightly more convenient for us not to do so.)

Definition 2.2. Let $\mathcal{V}$ and $\mathcal{W}$ be $\mathbb{S}$-modules. We define their direct $\operatorname{sum} \mathcal{V} \oplus \mathcal{W}$ componentwise and their tensor product by

$$
(\mathcal{V} \otimes \mathcal{W})(n)=\bigoplus_{k+l=n} \operatorname{Ind}_{\mathbb{S}_{k} \times \mathbb{S}_{l}}^{\mathbb{S}_{n}} \mathcal{V}(k) \otimes \mathcal{W}(l)
$$


This makes the category of $\mathbb{S}$-modules a symmetric monoidal category.

Definition 2.3. Let $\mathcal{V}$ and $\mathcal{W}$ be $\mathbb{S}$-modules. The plethysm $\mathcal{V} \circ \mathcal{W}$ is defined by

$$
(\mathcal{V} \circ \mathcal{W})(n)=\bigoplus_{k=1}^{\infty} \mathcal{V}(k) \otimes_{\mathbb{S}_{k}}\left(\mathcal{W}^{\otimes k}\right)(n)
$$

where $\left(\mathcal{W}^{\otimes k}\right)(n)$ is considered as an $\mathbb{S}_{k}$-module by permuting the factors, i.e. via the symmetric monoidal structure on $\mathbb{S}$-modules.

Let Ass denote the $\mathbb{S}$-module defined by

$$
\operatorname{Ass}(n)=\operatorname{Ind}_{\mathbb{Z} / n \mathbb{Z}}^{\mathbb{S}_{n}} \mathbf{1},
$$

where 1 is the trivial representation, i.e. the monoidal unit. Pictorially we think of $\operatorname{Ass}(n)$ as describing corollas with $n$ cyclically ordered input legs, or equivalently, with an embedding in the plane.

Proposition 2.4. The plethysm Ass $\circ \mathbf{a}_{0}^{\prime \prime}$ is the $\mathbb{S}$-module describing cyclically ordered necklaces of genus zero vertices.

Proof. Informally, we think of $\mathbf{a}_{0}^{\prime \prime}$ as corollas of genus zero with two marked legs. We think of the first as the "clockwise" one and the second as the "counterclockwise" one. There is an evident combinatorial bijection between cyclic necklaces of genus zero vertices and collections of genus zero vertices attached along two marked legs to a corolla with cyclically ordered inputs.

More formally, one can check from the definition of plethysm that one gets the correct result, using that

$$
\mathbf{a}_{0}^{\prime \prime}=\sum_{n \geq 3} \operatorname{ch} \operatorname{Res}_{\mathbb{S}_{n-2}}^{\mathbb{S}_{n}} \mathcal{V}((0, n))
$$

and that tensoring with $\operatorname{Ind}_{\mathbb{Z} / n \mathbb{Z}}^{\mathbb{S}_{n}} \mathbf{1}$ is the same as taking coinvariants under the action of $\mathbb{Z} / n \mathbb{Z}$.

Let $\Psi: \mathbb{S}_{n} \rightarrow \Lambda$ be the cycle map, defined as

$$
\Psi(x)=\prod_{\sigma \text { a cycle in } x} p_{|\sigma|} .
$$

Recall that $\Psi$ induces an isomorphism ch: $R\left(\mathbb{S}_{n}\right) \rightarrow \Lambda^{n}$ via

$$
V \mapsto \frac{1}{n !} \sum_{x \in \mathbb{S}_{n}} \operatorname{Tr}(x \mid V) \Psi(x) .
$$

Proposition 2.5. If $H$ is a subgroup of $\mathbb{S}_{n}$, then

$$
\operatorname{ch~Ind~}_{H}^{\mathbb{S}_{n}} \mathbf{1}=\frac{1}{H} \sum_{h \in H} \Psi(h) .
$$

Proof. See [Macdonald 1995, Chapter 1, Section 7, Example 4].

Proposition 2.6. There is an equality of generating series

$$
\sum_{n=1}^{\infty} \operatorname{ch} \operatorname{Ass}(n)=\sum_{n=1}^{\infty} \frac{1}{n} \sum_{d \mid n} \phi(d) p_{d}^{n / d}=-\sum_{n=1}^{\infty} \frac{\phi(n)}{n} \log \left(1-p_{n}\right) .
$$


Proof. The first equality follows from the preceding proposition, and the second by Taylor expanding and equating coefficients. See also [Getzler and Kapranov 1998, Example 7.6.2].

Proposition 2.7. The sum over all cyclically ordered necklaces is given by

$$
-\sum_{n \geq 1} \frac{\phi(n)}{n} \log \left(1-\psi_{n}\left(\mathbf{a}_{0}^{\prime \prime}\right)\right) .
$$

Proof. This follows now by putting together Propositions 2.4 and 2.6 ,

This is the formula needed in [Petersen 2012].

\section{NeCKLaCES AND WREath PRODUCtS}

A natural way to compute the sum over necklaces in a combinatorial fashion would be to interpret it, too, as a plethysm. One might let Dih denote the $\mathbb{S}$ module whose $n$th component is spanned by necklaces with $n$ vertices considered up to dihedral symmetry, i.e. the $\mathbb{S}_{n}$-module $\operatorname{Ind}_{D_{n}}^{\mathbb{S}_{n}} \mathbf{1}$, and then consider the plethysm $\operatorname{Dih} \circ \mathbf{a}_{0}^{\prime \prime}$.

This will however not give the right answer, and the basic problem with such an approach is that the action of the dihedral group on the dual graph of a necklace does not factor through the map $D_{n} \rightarrow \mathbb{S}_{n}$; indeed, $\mathbb{S}_{n}$ just acts by permuting the vertices, but the reflections in $D_{n}$ should act also by switching which of the two marked legs on each vertex should be "clockwise" and "counterclockwise".

To incorporate the possibility of having automorphisms which switch the two legs, we will have to work instead with the restriction

$$
\sum_{n \geq 3} \operatorname{Re}_{\mathbb{S}_{2} \times \mathbb{S}_{n-2}}^{\mathbb{S}_{n}} \mathcal{V}((0, n))
$$

and consider $D_{n}$ not as subgroup of $\mathbb{S}_{n}$ but of the hyperoctahedral group $\mathbb{S}_{2}$ 乙 $\mathbb{S}_{n}=$ $\left(\mathbb{S}_{2}\right)^{n} \rtimes \mathbb{S}_{n}$. Let $G$ be a finite group.

Definition 3.1. A $(G \times \mathbb{S})$-module $\mathcal{V}$ is a sequence $\mathcal{V}(n)$ of representations of $G \times \mathbb{S}_{n}$.

Definition 3.2. A $(G \prec \mathbb{S})$-module $\mathcal{W}$ is a sequence $\mathcal{W}(n)$ of representations of $G \curlywedge \mathbb{S}_{n}$.

Sums and tensor products of $(G \times \mathbb{S})$ - and $(G<\mathbb{S})$-modules are defined in the same way as for $\mathbb{S}$-modules.

Definition 3.3. Let $\mathcal{V}$ be a $(G \times \mathbb{S})$-module and $\mathcal{W}$ a $(G / \mathbb{S})$-module. We define the plethysm $\mathcal{W} \circ_{G} \mathcal{V}$ by

$$
\left(\mathcal{W} \circ_{G} \mathcal{V}\right)(n)=\bigoplus_{k \geq 0} \mathcal{W}(k) \otimes_{G \mathbb{S}_{k}}\left(\mathcal{V}^{\otimes k}\right)(n)
$$

Note that if $G$ acts on an object $V$ of a symmetric monoidal category, then $G$ ? $\mathbb{S}_{k}$ acts on $V^{\otimes k}$, so the tensor product above makes sense.

Remark 3.4. When $\mathcal{W}$ is concentrated in degree 1 , then $\mathcal{W}$ is just a representation of $G$ and we recover the ordinary tensor product of $G$-representations, i.e. $\mathcal{W}{ }^{\circ}{ }_{G} \mathcal{V}=$ $\mathcal{W} \otimes_{G} \mathcal{V}$ 
We consider the dihedral group $D_{n}$ as the subgroup of $\mathbb{S}_{2} \prec \mathbb{S}_{n}$ generated by the elements $(\mathbf{1}, \tau)$ and $(-\mathbf{1}, \sigma)$ where $\mathbf{1} \in \mathbb{S}_{2}^{n}$ is the element $(1,1, \ldots, 1), \mathbf{- 1}$ is the element $(-1,-1, \ldots,-1), \tau \in \mathbb{S}_{n}$ is the $n$-cycle $(12 \cdots n)$, and $\sigma$ is the reflection $(1 n)(2, n-1) \cdots$.

Definition 3.5. Let Dih be the $\left(\mathbb{S}_{2} 2 \mathbb{S}\right)$-module defined by $\operatorname{Dih}(n)=\operatorname{Ind}_{D_{n}}^{\mathbb{S}_{2} \mathbb{S}_{n}} \mathbf{1}$.

Definition 3.6. For an $\mathbb{S}$-module $\mathcal{V}$, let $\mathcal{V}^{(n)}$ denote its restriction to an $\left(\mathbb{S}_{n} \times \mathbb{S}\right)$ module.

Proposition 3.7. The underlying $\mathbb{S}$-module of the $\left(\mathbb{S}_{2} \times \mathbb{S}\right)$-module

$$
\operatorname{Dih} \circ_{\mathbb{S}_{2}} \mathbf{a}_{0}^{(2)}
$$

is the submodule of $\mathbf{b}_{1}$ of unordered necklaces of genus zero vertices.

Proof. The proof is now the same as the proof of Proposition 2.4.

To describe the $\mathbb{S}_{2}$ 乙 $\mathbb{S}$-module Dih, we shall need to work with the $\operatorname{ring} \Lambda(G)$ of wreath product symmetric functions. This ring is defined in [Macdonald 1995, Chapter I, Appendix B]. The ring $\Lambda(G)$ is generated as an algebra by generalized power sums $p_{n}(c)$ where $n$ is a positive integer and $c$ is a conjugacy class of $G$. The degree of $p_{n}(c)$ is $n$. There is a natural map

$$
\Psi: G \prec \mathbb{S}_{n} \rightarrow \Lambda(G)^{n}
$$

generalizing the cycle map $\mathbb{S}_{n} \rightarrow \Lambda^{n}$. One computes $\Psi\left(g_{1}, \ldots, g_{n}, x\right)$ as follows: for each cycle $\sigma$ of $x$, take the product of the corresponding $g_{i}$; this product lies in a well-defined conjugacy class $c(\sigma)$ of $G$. Then

$$
\Psi\left(g_{1}, \ldots, g_{n}, x\right)=\prod_{\sigma \text { a cycle in } x} p_{|\sigma|}(c(\sigma)) .
$$

As before there is an isomorphism onto the degree $n$ part, ch: $R\left(G \prec \mathbb{S}_{n}\right) \rightarrow \Lambda(G)^{n}$, defined by

$$
V \mapsto \frac{1}{|G|^{n} n !} \sum_{x \in G i \mathbb{S}_{n}} \operatorname{Tr}(x \mid V) \Psi(x) .
$$

The plethysm of $(G<\mathbb{S})$-modules and $(G \times \mathbb{S})$-modules can now be described equivalently as an action of $\Lambda(G)$ on $R(G) \otimes \Lambda$.

Proposition 2.5 holds true for wreath product symmetric functions - the proof given in Macdonald's book carries over without changes. Hence we have:

Proposition 3.8. Let $H$ be a subgroup of $G<\mathbb{S}_{n}$. Then

$$
\operatorname{Ind}_{H}^{G i \mathbb{S}_{n}} \mathbf{1}=\frac{1}{|H|} \sum_{h \in H} \Psi(h) \in \Lambda(G) .
$$

Proposition 3.9. Let $G=\mathbb{S}_{2}$, and denote the power sums in $\Lambda\left(\mathbb{S}_{2}\right)$ corresponding to the identity conjugacy class by $\mathfrak{p}_{n}$ and the power sums corresponding to the nonidentity by $\mathfrak{q}_{n}$. Then

$$
\sum_{n \geq 1} \operatorname{ch} \operatorname{Dih}(n)=-\frac{1}{2} \sum_{n \geq 1} \frac{\phi(n)}{n} \log \left(1-\mathfrak{p}_{n}\right)+\frac{\frac{\mathfrak{q}_{1}}{2}\left(1+\frac{\mathfrak{q}_{1}}{2}\right)+\frac{1}{4} \mathfrak{p}_{2}}{1-\mathfrak{p}_{2}}
$$


Proof. From Proposition 3.8 and the definition of $\Psi$ one sees that

$$
\operatorname{ch~} \operatorname{Ind}_{D_{n}}^{\mathbb{S}_{2} \mathbb{S}_{n}} \mathbf{1}=\frac{1}{2 n} \sum_{d \mid n} \phi(d) \mathfrak{p}_{d}^{n / d}+ \begin{cases}\frac{1}{4}\left(\mathfrak{q}_{1}^{2} \mathfrak{p}_{2}^{n / 2-1}+\mathfrak{p}_{2}^{n / 2+1}\right) & n \text { even } \\ \frac{1}{2} \mathfrak{q}_{1} \mathfrak{p}_{2}^{(n-1) / 2} & n \text { odd }\end{cases}
$$

where the first term is the sum over all rotations in $D_{n}$ and the second is the sum over all reflections. Comparing this with the result of Taylor expanding the logarithms and the geometric series gives the result.

Proposition 3.10. Let $G=\mathbb{S}_{n}$. The isomorphism $\Lambda\left(\mathbb{S}_{n}\right)^{1} \rightarrow \Lambda^{n}$ is given as follows: if the conjugacy class $c$ in $\mathbb{S}_{n}$ is given by the cycle type $\left(\lambda_{1}, \ldots, \lambda_{k}\right) \vdash n$, then $p_{1}(c) \mapsto p_{\lambda_{1}} \cdots p_{\lambda_{k}}$.

Proof. One needs only to compare the different isomorphisms

$$
\Lambda^{n} \leftarrow R\left(\mathbb{S}_{n}\right)=R\left(\mathbb{S}_{n}\left\langle\mathbb{S}_{1}\right) \rightarrow \Lambda\left(\mathbb{S}_{n}\right)^{1} .\right.
$$

For a symmetric function $f\left(p_{1}, p_{2}, \ldots\right) \in \Lambda$, let $D(f)=f\left(\frac{\partial}{\partial p_{1}}, 2 \frac{\partial}{\partial p_{2}}, \ldots\right)$.

Proposition 3.11. Let $f \in \Lambda^{k}=\Lambda\left(\mathbb{S}_{k}\right)^{1}$ and $g \in \Lambda$. Then

$$
f \circ \circ_{\mathbb{S}_{k}} g^{(k)}=D(f) g \text {. }
$$

Proof. Suppose $\operatorname{ch} U=f$ and $\operatorname{ch} V=g \in \Lambda^{n+k}$. Then

$$
U \circ_{\mathbb{S}_{k}} V^{(k)}=U \otimes_{\mathbb{S}_{k}} V^{(k)}=\operatorname{Hom}_{\mathbb{S}_{k}}\left(U, \operatorname{Res}_{\mathbb{S}_{k} \times \mathbb{S}_{n}}^{\mathbb{S}_{n+k}} V\right)
$$

by Remark 3.4 and since all representations of $\mathbb{S}_{k}$ are self-dual. The characteristic of the latter is equal to the right hand side by [Getzler and Kapranov 1998, p. 8.10]. One easily extends the result to virtual representations and non-homogeneous $g$.

Proposition 3.12. One has that $\mathfrak{p}_{n} \circ_{\mathbb{S}_{2}} f^{(2)}=\psi_{n}\left(f^{\prime \prime}\right)$, and $\mathfrak{q}_{n} \circ_{\mathbb{S}_{2}} f^{(2)}=2 \psi_{n}(\dot{f})$.

Proof. Suppose first that $n=1$. Then $\mathfrak{p}_{1}$ and $\mathfrak{q}_{1}$ in $\Lambda\left(\mathbb{S}_{2}\right)^{1}$ correspond to $p_{1}^{2}$ and $p_{2}$ in $\Lambda^{2}$ by Proposition 3.10, so by Proposition 3.11 we have

$$
\mathfrak{p}_{1} \circ_{\mathbb{S}_{2}} f^{(2)}=D\left(p_{1}^{2}\right) f=f^{\prime \prime}
$$

and

$$
\mathfrak{q}_{1} \circ_{\mathbb{S}_{2}} f^{(2)}=D\left(p_{2}\right) f=2 \dot{f} \text {. }
$$

In general one has $\mathfrak{p}_{n} \circ_{\mathbb{S}_{2}} f^{(2)}=p_{n} \circ \mathfrak{p}_{1} \circ_{\mathbb{S}_{2}} f^{(2)}=\psi_{n}\left(f^{\prime \prime}\right)$, and $\mathfrak{q}_{n} \circ_{\mathbb{S}_{2}} f^{(2)}=$ $p_{n} \circ \mathfrak{q}_{1} \circ_{\mathbb{S}_{2}} f^{(2)}=2 \psi_{n}(\dot{f})$. The associativity and the $\lambda$-ring structure on $\Lambda(G)$ used here is most easily seen from the interpretation as polynomial functors, cf. [Macdonald 1980].

Theorem 3.13. The sum

$$
-\frac{1}{2} \sum_{n \geq 1} \frac{\phi(n)}{n} \log \left(1-\psi_{n}\left(\mathbf{a}_{0}^{\prime \prime}\right)\right)+\frac{\dot{\mathbf{a}}_{0}\left(1+\dot{\mathbf{a}}_{0}\right)+\frac{1}{4} \psi_{2}\left(\mathbf{a}_{0}^{\prime \prime}\right)}{1-\psi_{2}\left(\mathbf{a}_{0}^{\prime \prime}\right)}
$$

computes the characteristic of the submodule of $\mathbb{M V}$ spanned by necklaces of genus zero vertices.

Proof. This follows by putting together Propositions 3.7, 3.9 and 3.12. 


\section{REFERENCES}

Consani, Caterina and Faber, Carel (2006). "On the cusp form motives in genus 1 and level 1". Moduli spaces and arithmetic geometry. Vol. 45. Adv. Stud. Pure Math. Tokyo: Math. Soc. Japan, 297-314.

Getzler, Ezra (1995). "Operads and moduli spaces of genus 0 Riemann surfaces". The moduli space of curves (Texel Island, 1994). Vol. 129. Progr. Math. Boston, MA: Birkhäuser Boston, 199-230.

Getzler, Ezra (1998). "The semi-classical approximation for modular operads". Comm. Math. Phys. 194 (2), 481-492.

Getzler, Ezra and Kapranov, Mikhail M. (1998). "Modular operads". Compositio Math. 110 (1), 65-126.

Macdonald, Ian G. (1980). "Polynomial functors and wreath products". J. Pure Appl. Algebra 18 (2), 173-204.

Macdonald, Ian G. (1995). Symmetric functions and Hall polynomials. Second. Oxford Mathematical Monographs. With contributions by A. Zelevinsky, Oxford Science Publications. New York: The Clarendon Press Oxford University Press, $\mathrm{x}+475$ pages.

Petersen, Dan (2012). "Cusp form motives and admissible G-covers". Algebra Number Theory 6 (6), 1199-1221. 